\title{
Microscopic expressions for the rigidity constants of a simple liquid-vapor interface
}

Cite as: J. Chem. Phys. 95, 6986 (1991); https://doi.org/10.1063/1.461509

Submitted: 18 July 1991 . Accepted: 19 August 1991 . Published Online: 31 August 1998

Edgar M. Blokhuis, and Dick Bedeaux

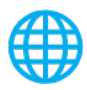

\section{ARTICLES YOU MAY BE INTERESTED IN}

Pressure tensor of a spherical interface

The Journal of Chemical Physics 97, 3576 (1992); https://doi.org/10.1063/1.462992

Determination of curvature corrections to the surface tension of a liquid-vapor interface through molecular dynamics simulations

The Journal of Chemical Physics 116, 302 (2002); https://doi.org/10.1063/1.1423617

The Statistical Mechanical Theory of Surface Tension

The Journal of Chemical Physics 17, 338 (1949); https://doi.org/10.1063/1.1747248

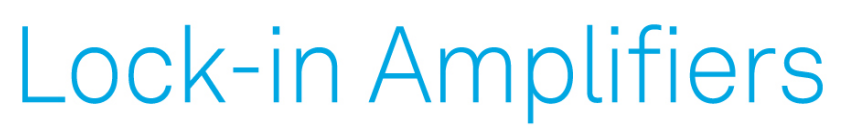

... and more, from DC to $600 \mathrm{MHz}$

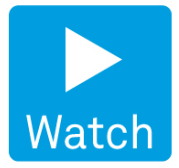




\title{
LETTERS TO THE EDITOR
}

The Letters to the Editor section is divided into four categories entitled Communications, Notes, Comments, and Errata. Communications are limited to three and one half journal pages, and Notes, Comments, and Errata are limited to one and three-fourths journal pages as described in the Announcement in the I July 1991 issue.

\section{COMMUNICATIONS}

\section{Microscopic expressions for the rigidity constants of a simple liquid-vapor interface}

\author{
Edgar M. Blokhuis and Dick Bedeaux \\ Department of Physical and Macromolecular Chemistry, Gorlaeus Laboratories, P. O. Box 9502, \\ 2300 RA Leiden, The Netherlands
}

(Received 18 July 1991; accepted 19 August 1991)

Of late there has been a growing interest in the understanding of curved interfaces. In particular, attention has focused on the influence of the rigidity constant of bending, $k$, and the rigidity constant associated with Gaussian curvature $\vec{k}$, on the behavior of interfaces. For some properties of simple liquid interfaces these rigidity constants play a significant role. ${ }^{1,2}$ In systems where for some reason the surface tension is small these rigidity constants even become the dominant factor in understanding both the static and dynamic behavior of interfaces. In this communication we present expressions for the rigidity constants of an interface by calculating the change in surface free energy under transformations which change both the surface area and radius of curvature but leave the volume of the system unchanged. ${ }^{3}$ The resulting equations are analogous to the well-known microscopic expression for the surface tension derived in 1949 by Kirkwood and Buff. ${ }^{4}$ The formulas are subsequently simplified using an approximate expression for the curvature dependent density autocorrelation function near the interface. Explicit expressions for the rigidity constants far from the critical point as well as close to the critical point are given as integrals over the product of the interaction potential and the pair correlation function in the liquid phase. Values for the rigidity constants close to the critical point are calculated.

The isothermal change in surface free energy of a spherical interface with radius $R$ is given by ${ }^{5}$

$$
d F_{s}=\sigma(R) d A+C(R) A d R \quad \text { (sphere), }
$$

where $\sigma(R)$ and $C(R)$ are the radius dependent surface tension and curvature term. In order for this expression to be the only contribution to the surface free energy we have chosen the position of the dividing surface such that there is no adsorption at the interface so that the total number of particles, $N$, equals $\rho_{l} V_{l}+\rho_{g} V_{g}$ with $\rho_{l, g}$ and $V_{l, g}$ the density and volume of the liquid, gas phase. The surface free energy for a generally curved interface is given by ${ }^{6}$

$$
F_{s}=\int d A\left[f_{0}+\frac{k}{2}\left(C_{1}+C_{2}-C_{0}\right)^{2}+\bar{k} C_{1} C_{2}\right]
$$

where $C_{1}$ and $C_{2}$ are the principal radii of local curvature. Equation (2) is an expansion to second order in the curvature and defines the coefficients $f_{0}, C_{0}, k$, and $\bar{k}$. The coefficient $f_{0}$ is related to the surface tension by $f_{0}$ $+(k / 2) C_{0}^{2}=\sigma$ and $C_{0}$ is the spontaneous curvature of the interface. For a sphere, where $C_{1}=C_{2}=-1 / R$, we obtain for the radius dependent surface tension and curvature term using the above equation:

$$
\begin{aligned}
\sigma(R) & =\sigma+2 k C_{0} \frac{1}{R}+(2 k+\bar{k})\left(\frac{1}{R}\right)^{2}, \\
C(R) & =\left.\frac{\partial \sigma(R)}{\partial R}\right|_{T} \quad \text { (sphere) } \\
& =-2 k C_{0}\left(\frac{1}{R}\right)^{2}-2(2 k+\bar{k})\left(\frac{1}{R}\right)^{3} .
\end{aligned}
$$

For a one-component two-phase system in the canonical ensemble where the total configurational energy is the sum of pair potentials the change in surface free energy generated by an arbitrary infinitesimal coordinate transformation $\mathbf{r} \rightarrow \mathbf{r}+\delta \mathbf{r}$ is given by

$\delta F_{s}=\frac{1}{2} \int d \mathbf{r}_{1} \int d \mathbf{r}_{2} \rho^{(2)}\left(\mathbf{r}_{1}, \mathbf{r}_{2}\right) u^{\prime}(r) \frac{\mathbf{r}_{12}}{r} \cdot\left(\delta \mathbf{r}_{2}-\delta \mathbf{r}_{1}\right)$

with $\rho^{(2)}\left(\mathbf{r}_{1}, \mathbf{r}_{2}\right)$ the density auto correlation function, $\mathbf{r}_{12} \equiv \mathbf{r}_{2}-\mathbf{r}_{1}, r \equiv\left|\mathbf{r}_{12}\right|$ and $u^{\prime}(r)$ is the derivative with respect to its argument of the pair interaction potential. To avoid bulk contributions to $\delta F$ we consider coordinate transformations which leave the bulk volumes unchanged. By calculating the change in free energy for two such transformations we derive the following expressions for $\sigma(R)$ and $C(R)$ : 


$$
\begin{aligned}
& \sigma(R)=\frac{1}{4} \int d z_{1} \int d \mathbf{r}_{12} u^{\prime}(r) r \rho_{s}^{(2)}\left(z_{1}, z_{2}, r\right)\left[\left(1-3 s^{2}\right)\left(1+\frac{z_{1} z_{2}}{R^{2}}\right)+\frac{r^{2} s^{2}}{3 R^{2}}\left(3-5 s^{2}\right)\right], \\
& C(R)=\frac{1}{4 R} \int d z_{1} \int d \mathbf{r}_{12} u^{\prime}(r) r \rho_{s}^{(2)}\left(z_{1}, z_{2}, r\right)\left[-\left(1-3 s^{2}\right)\left(\frac{z_{1}+z_{2}}{R}\right)+\frac{r^{2} s^{2}}{3 R^{2}}\left(3-5 s^{2}\right)\right],
\end{aligned}
$$

where $s \equiv \cos \theta_{12}, \quad z_{2} \equiv z_{1}+s r$ and we have used the spherical symmetry of the system to write $\rho^{(2)}\left(\mathbf{r}_{1}, \mathbf{r}_{2}\right)$ $=\rho s(2)(|\mathbf{r} 1|,|\mathrm{r} 2|, r)$. In order to obtain expressions for $k$ and $\bar{k}$ separately we perform an analogous calculation for a cylindrical interface. With $C_{1}=-1 / R$ and $C_{2}=0 \mathrm{Eq}$. (2) yields

$$
\begin{aligned}
& \sigma(R)=\sigma+k C_{0} \frac{1}{R}+\frac{k}{2}\left(\frac{1}{R}\right)^{2}, \\
& C(R)=\left.\frac{\partial \sigma(R)}{\partial R}\right|_{T}=-k C_{0}\left(\frac{1}{R}\right)^{2}-k\left(\frac{1}{R}\right)^{3} .
\end{aligned}
$$

Again using Eq. (4) we derive for the cylinder

$$
\begin{aligned}
& \sigma(R)=\frac{1}{4} \int d z_{1} \int d \mathrm{r}_{12} u^{\prime}(r) r \rho_{c}^{(2)}\left(z_{1}, z_{2}, r\right)\left[\left(1-3 s^{2}\right)\left(1+\frac{z_{1} z_{2}}{2 R^{2}}\right)+\frac{r^{2}}{32 R^{2}}\left(1+6 s^{2}-15 s^{4}\right)\right] \\
& C(R)=\frac{1}{4 R} \int d z_{1} \int d \mathbf{r}_{12} u^{\prime}(r) r \rho_{c}^{(2)}\left(z_{1}, z_{2}, r\right)\left[-\left(1-3 s^{2}\right)\left(\frac{z_{1}+z_{2}}{R}-\frac{z_{1} z_{2}}{2 R^{2}}\right)-\frac{r^{2}}{16 R^{2}}\left(1+6 s^{2}-15 s^{4}\right)\right] .
\end{aligned}
$$

Identifying the coefficients in the expansion in $1 / R$ with the thermodynamical quantities $\sigma, k C_{0}, k$ and $\bar{k}$ by comparing Eqs. (5) and (7) with Eqs. (3) and (6), respectively, one obtains the following formulas:

$$
\begin{aligned}
\sigma= & \frac{1}{4} \int d z_{1} \int d \mathbf{r}_{12} u^{\prime}(r) r\left(1-3 s^{2}\right) \rho_{f}^{(2)} \\
k C_{0}= & \frac{1}{4} \int d z_{1} \int d \mathbf{r}_{12} u^{\prime}(r) r\left(1-3 s^{2}\right) \frac{1}{2} \rho_{s, 1}^{(2)}, \\
k C_{0}= & \frac{1}{4} \int d z_{1} \int d \mathbf{r}_{12} u^{\prime}(r) r\left(1-3 s^{2}\right) \rho_{c, 1}^{(2)}, \\
k C_{0}= & \frac{1}{4} \int d z_{1} \int d \mathbf{r}_{12} u^{\prime}(r) r\left(1-3 s^{2}\right) \frac{z_{1}+z_{2}}{2} \rho_{f}^{(2)} \\
k= & \frac{1}{4} \int d z_{1} \int d \mathbf{r}_{12} u^{\prime}(r) r\left[\left(1-3 s^{2}\right)\right. \\
& \left.\times\left(2 \rho_{c, 2}^{(2)}+z_{1} z_{2} \rho_{f}^{(2)}\right)+\frac{r^{2}}{16}\left(1+6 s^{2}-15 s^{4}\right) \rho_{f}^{(2)}\right] \\
k= & \frac{1}{4} \int d z_{1} \int d \mathbf{r}_{12} u^{\prime}(r) r\left[( 1 - 3 s ^ { 2 } ) \left(\frac{z_{1}+z_{2}}{2} \rho_{c, 1}^{(2)}\right.\right. \\
& \left.\left.-\frac{z_{1} z_{2}}{2} \rho_{f}^{(2)}\right)-\frac{r^{2}}{16}\left(1+6 s^{2}-15 s^{4}\right) \rho_{f}^{(2)}\right] \\
\bar{k}= & \frac{1}{4} \int d z_{1} \int d \mathbf{r}_{12} u^{\prime}(r) r\left[( 1 - 3 s ^ { 2 } ) \left(\rho_{s, 2}^{(2)}-4 \rho_{c, 2}^{(2)}\right.\right. \\
& \left.\left.-2 z_{1} z_{2} \rho_{f}^{(2)}\right)-\frac{r^{2}}{24}\left(3-6 s^{2}-5 s^{4}\right) \rho_{f}^{(2)}\right] \\
\bar{k}= & \frac{1}{4} \int d z_{1} \int d \mathbf{r}_{12} u^{\prime}(r) r\left[\left(1-3 s^{2}\right)\left(\frac{z_{1}+z_{2}}{2}\left[\rho_{s, 1}^{(2)}\right]+z_{1} z_{2} \rho_{f}^{(2)}\right)+\frac{r^{2}}{24}\left(3-6 s^{2}-5 s^{4}\right) \rho_{f}^{(2)}\right]
\end{aligned}
$$

where we have also expanded the correlation functions for the spherical and cylindrical interface up to second order in the inverse radius

$$
\begin{aligned}
& \rho_{s}^{(2)}=\rho_{f}^{(2)}+\rho_{s, 1}^{(2)}\left(\frac{1}{R}\right)+\rho_{s, 2}^{(2)}\left(\frac{1}{R}\right)^{2}, \\
& \rho_{c}^{(2)}=\rho_{f}^{(2)}+\rho_{c, 1}^{(2)}\left(\frac{1}{R}\right)+\rho_{c, 2}^{(2)}\left(\frac{1}{R}\right)^{2} .
\end{aligned}
$$

Here $\rho_{f}^{(2)}$ is the pair correlation function of the flat interface. For notational convenience we have left out the explicit dependence on $z_{1}, z_{2}$ and $r$ of the correlation functions in the above equations. The expression for $\sigma$ is the well-known Kirkwood-Buff formula. The other expressions are analogous rigorous expressions for $k C_{0}, k$ and $\bar{k}$ in terms of correlation functions. For $k C_{0}$ it is sufficient to know the correlation function of the flat interface and for $k$ and $\bar{k}$ one needs the modification of the correlation functions to first order in the inverse radius for the spherical and cylindrical interface. An unpleasant feature is the fact that not much is known about the curvature dependence of the correlation function. Using the equivalence of the three expressions for $k C_{0}$ we postulate the following relation which makes these expressions identical:

$$
\rho_{s, 1}^{(2)}=2 \rho_{c, 1}^{(2)}=\left(z_{1}+z_{2}\right) \rho_{f}^{(2)} .
$$

We have not been able to find a more fundamental justification of this formula.

For the auto correlation function of the flat interface we assume the following simple form:

$$
\rho_{f}^{(2)}\left(z_{1}, z_{2}, r\right)=\rho\left(z_{1}\right) \rho\left(z_{2}\right) g(r),
$$

where $g(r)$ is the pair correlation function in the uniform liquid. Close to the critical point the pair correlation function in the liquid and vapor region become identical so that 
this is a reasonable approximation. Far from the critical point this approximation, though widely used, ${ }^{5,7}$ is more questionable. For the density profile we first consider the well-known van der Waals form

$$
\rho(z)=\rho_{c}-\frac{\Delta \rho}{2} \tanh (z / 2 \xi),
$$

where $\rho_{c}=\frac{1}{2}\left(\rho_{l}+\rho_{g}\right), \Delta \rho=\left(\rho_{l}-\rho_{g}\right)$ and $\xi$ the bulk correlation length which is a measure of the thickness of the interface. From the above equations one finds the following.

Far from the critical point $(\xi \rightarrow 0)$

$$
\begin{aligned}
& \sigma=\frac{\pi}{8} \Delta \rho^{2} \int d r r^{4} u^{\prime}(r) g(r), \\
& k=\frac{\pi}{192} \Delta \rho^{2} \int d r r^{6} u^{\prime}(r) g(r), \\
& \bar{k}=\frac{\pi}{288} \Delta \rho^{2} \int d r r^{6} u^{\prime}(r) g(r) .
\end{aligned}
$$

The first equation is the well-known Fowler formula for the surface tension. ${ }^{7}$

Close to the critical point $(\xi \rightarrow \infty)$

$$
\begin{aligned}
& \sigma=\frac{\pi}{45} \frac{\Delta \rho^{2}}{\xi} \int d r r^{5} u^{\prime}(r) g(r), \\
& k=\frac{\pi}{270}\left(\pi^{2}+12\right) \Delta \rho^{2} \xi \int d r r^{5} u^{\prime}(r) g(r), \\
& \bar{k}=\frac{\pi}{135}\left(\pi^{2}-6\right) \Delta \rho^{2} \xi \int d r r^{5} u^{\prime}(r) g(r) .
\end{aligned}
$$

These last equations lead to the following expression for the critical rigidity constants:

$$
k=\frac{1}{2} \frac{\pi^{2}+12}{\pi^{2}-6} \bar{k}=\left(\frac{\pi^{2}}{6}+2\right) \sigma \xi^{2}
$$

The ratio $R_{\sigma} \equiv \sigma \xi^{2} / k_{B} T_{c}$ is a universal constant for the surface tension near the critical point. Experimentally one finds $R_{\sigma}=0.10$ while on the basis of renormalization group theory one calculates $R_{\sigma}=0.128 .^{8,9}$ The commonly accepted explanation for this difference is that capillary waves are not incorporated in the renormalization group calculation. ${ }^{9}$ Using the tanh profile and $R_{\sigma}=0.128$ we find as critical rigidity constants $k=0.467 k_{B} T_{c}$ and $k=0.165$ $k_{B} T_{c}$. Close to the critical point the Fisk-Widom profile ${ }^{10}$ is more appropriate than the tanh profile. The critical rigidity constants are then found to be $k=0.631 k_{B} T_{c}$ and $k=0.239 k_{B} T_{c}$ where again $R_{\sigma}=0.128$ has been used.

As the approximation in Eq. (11) is expected to hold near the critical point the critical rigidity constants are in fact found to be universal constants, $R_{k}$ and $R_{\bar{k}}$, times $k_{B} T_{c}$ provided that also Eq. (10) is valid for the dominant contribution in the critical point. The analysis thus seems to suggest universal values of $R_{k}$ and $R_{\bar{k}}$. An analysis of ellipsometric data for binary liquids near the critical point gives a value $R_{k}=1.1^{2}$ which, though being of the same order of magnitude, is clearly larger than the largest theoretical value $R_{k}=0.631$. A more thorough calculation of $R_{k}$ and $R_{\bar{k}}$ would require a better understanding of the behavior of the density autocorrelation function in the vicinity of the interface and in particular of the contribution due to capillary waves. The contribution of capillary waves to $R_{k}$ is expected to increase $R_{k}^{11}$ and may therefore help to reduce this discrepancy.

This work is part of the research program of the Leiden Material Science Center and of the "Stichting voor Fundamenteel Onderzoek der Materie" (FOM).

\footnotetext{
'J. Meunier, J. Physique 48, 1819 (1987).

${ }^{2}$ E. M. Blokhuis and D. Bedeaux, Physica A 164, 515 (1990).

${ }^{3}$ Details of the calculation will be given in a future publication.

${ }^{4}$ J. G. Kirkwood and F. P. Buff, J. Chem. Phys. 17, 338 (1949); F. P. Buff, ibid. 23, 419 (1955).

${ }^{5}$ J. S. Rowlinson and B. Widom, Molecular Theory of Capillarity (Clarendon, Oxford, 1982).

${ }^{6}$ W. Helfrich, Z. Naturforsch. 28c, 693 (1973).

${ }^{7}$ R. H. Fowler, Proc. R.Soc. A 159, 229 (1937).

${ }^{8}$ M. R. Moldover, Phys. Rev. A 31, 1022 (1985).

${ }^{9}$ J. V. Sengers ad J. M. J. van Leeuwen, Phys. Rev A 39, 6346 (1989).

${ }^{10} \mathrm{~S}$. Fisk and B Widom, J. Chem. Phys. 50, 3219 (1969).

"B. P. Binks, J. Meunier, O. Abillon, and D. Langevin, Langmuir 5, 415 (1989).
} 